\title{
The Volkmann dogma: a retrospective, long-term, single-center study
}

\author{
D. Heim $\cdot$ K. Niederhauser $\cdot$ N. Simbrey
}

Received: 17 August 2010/Accepted: 2 November 2010/Published online: 24 November 2010

(C) Urban \& Vogel 2010

\begin{abstract}
Introduction There has been (and still is) some controversy about the treatment of malleolar fractures with an additional fracture of the posterior malleolus (Volkmann triangle) in the last few years. Should a posterior malleolus with a fragment that is larger than $25 \%$ of the articular surface be fixed? Can long-term results be influenced by the fixation of the Volkmann triangle?

Materials and methods In a consecutive series of 43/56 patients with a dislocated malleolar fracture, clinical and radiological results were analyzed after a follow-up of 7.3 years (4-11 years). There were 15 men with an average age of 53 years and 28 women with an average age of 62 years, respectively. Eleven fragments were fixed. At inspection, 16 patients displayed a cartilage lesion at the talar dome.

Results No or only slight arthrosis was found on X-rays of 37/43 patients. Six patients showed moderate or severe arthrosis. The Olerud-Molander score was 95.5 in patients without a Volkmann triangle, 98.5 in the presence of a small, unfixed fragment, and 90.6 with a large fixed fragment. Patients with no cartilage damage on perioperative inspection presented less severe arthrosis in the long-term results, while those with documented cartilage damage
\end{abstract}

D. Heim $(\bowtie)$

Chefarzt Chirurgie, Spital Frutigen, Adelbodenstr,

3714 Frutigen, Switzerland

e-mail: dominik.heim@spitalfmi.ch

K. Niederhauser

Department of Gynecology and Obstetrics, Inselspital,

3000 Bern, Switzerland

N. Simbrey

Department of Thoracic Surgery, Inselspital,

3000 Bern, Switzerland during the operation had more severe arthrosis. The Olerud-Molander score was 97.2 without cartilage damage and 94.5 with cartilage damage.

Conclusion In conclusion, the presence of a Volkmann triangle impairs prognosis; fixation, even with anatomic results, does not return the score to "normal." Cartilage damage seems to play a crucial role in the long-term outcome.

Keywords Malleolar fractures · Volkmann triangle . Cartilage damage

\section{Introduction}

In 1940, Nelson and Jensen [1] published their results on malleolar fractures with fracture of the so-called Volkmann triangle (posterior malleolus). They stated, based on their small series of eight patients, that fragments that were bigger than one-third of the articular surface and were fixed did better than those without fixation. Even seventy years after they published their results, we still respect this rule. Some years later, McLaughlin [2] reduced the size of the fragment that should be fixed to larger than one-quarter of the articular surface. In later series [3, 4], these findings were confirmed in that sense that fractures with an additional Volkmann triangle led to an impaired prognosis $[3,5]$, while fractures with bigger fragments that were fixed yielded a better prognosis than those that were unfixed [3]. On the other hand, fragments that were smaller than this played a less important role [6]. Two issues were attributed to the size of the Volkmann triangle that predisposed for subsequent arthrosis: a decrease in contact area [7] and the tendency for posterior subluxation $[8,9]$. It was stated that "in cases of larger fragments with permanent displacement 
... there is another trigger factor for later osteoarthritis besides articular incongruency, namely instability with talar subluxation" [3].

The aim of our study was to determine the long-term radiological results of malleolar fractures with an additional Volkmann fragment and to assess the role of a fracture of the posterior malleolus on arthrosis. Does a fracture of the posterior malleolus play such a crucial role in ankle fractures as described in Nelson's paper, or are other factors responsible for the the long-term outcome? To answer these questions, the worst case scenario was chosen. The investigation focused solely on dislocated malleolar fractures. All other displaced fractures (but not dislocated/luxated) were excluded. A single-center study was performed on a prospective database with a retrospective review.

\section{Materials and methods}

Since 1995 all of the fractures that have been admitted to our hospital and have received surgical treatment (internal or external) have been recorded prospectively. All patients are called in for a one-year check-up, including clinical and X-ray checks. All patients are checked personally by their respective surgeon. Further surgery (e.g., implant removal) or definitive dismissal is discussed routinely with the patient at this occasion.

For the present study, all patients with a dislocated malleolar fracture were invited for a further check, including a physical and a radiological examination, at a follow-up of at least 4 years. The clinical findings were obtained and recorded by a Ph.D. student under the supervision of the head of the investigation (D.H.), and the $\mathrm{X}$-rays were analyzed by an independent radiologist.

Arthrosis was graded between 0 and 4 in a slightly modified way according to the classification of Knirk and Jupiter [10], with grade 0 signifying no arthrosis, grade 1 representing sclerosis of the articular surface, grade 2 indicating the formation of cysts and osteophytes, grade 3 suggesting a narrowing of the joint, and grade 4 pointing to ankylosis of the joint.

Statistical comparisons of scores in two or more groups were performed with Student's $t$ test or one way analysis of variance (ANOVA), respectively.

\section{Results}

Fifty-six patients met the abovementioned criteria and were included in this retrospective study. It was possible to follow 43 (76\%) of these 56 patients: this included 15 men (31\%) with an average age of 53 years and 28 women (69\%) with an average age of 62 years. Twenty-four patients had a broken left ankle, and 19 had a right ankle break. Thirteen patients were lost to follow-up, 2 died of causes unrelated to the fracture, 8 patients were lost in their respective countries, and 3 refused to undergo another examination as they were satisfied with their results. The average time to follow-up was 7.3 years (3.9-10.9 years).

Fractures were classified into bi- or trimalleolar fractures and according to the classification of Weber [11] into $\mathrm{A}, \mathrm{B}$, and $\mathrm{C}$. Considering the small series of fractures, we did not use the much more extensive AO classification. Among the fractures followed, 9 were bi- and 34 were trimalleolar fractures. There were 23 Weber type B fractures and 20 type $\mathrm{C}$ fractures. Four fractures were open fractures, 3 were grade 1 and 1 was grade 2 according to the Gustilo-Anderson classification [12]. A cartilage lesion of the talar dome was diagnosed in 16 patients during the surgical intervention. An arthroscopy and/or a CT scan was not carried out prior to the internal fixation.

Presence and surgery of a Volkmann triangle

Of the 43 dislocated and followed fractures registered, 7 had no Volkmann triangle, 10 had just a very small shelllike fragment, 18 had a fragment smaller than one-quarter of the articular surface, and 8 fractures had a fragment that was bigger than this; these 8 fragments were all fixed. Four fragments were fixed by an anteroposterior lag screw, one lag screw was added from posterior, and three fractures were fixed with a one-third tubular antiglide plate on the posterior part of the distal tibia. In the case of residual instability upon the completion of internal fixation, an additional positioning screw was used. Most fractures were operated on primarily.

\section{Complications}

There were no infections and in all cases the recovery of the soft tissues upon discharge was uneventful. There was one malunion 6 weeks after fixation with an ap screw, which was corrected by re-reduction and fixation with two lag screws from posterior. One antiglide plate had to removed early after 6 months due to patient discomfort; the removal of the implant was followed by a disturbance of the posterior tibial nerve.

Results immediately after internal fixation

Anatomical reduction of a small Volkmann triangle by internal fixation of the external and internal malleoli happened in 8/18 patients, $10 / 18$ fragments did not return to their anatomical positions. No attention was paid to the shell-like fragments. All 8 fragments that were larger than one-quarter of the articular surface were fixed in the 
abovementioned manner. Two of the 11 fragments were fixed with a slight step-off of $1 \mathrm{~mm}$ (one screw from anterior and one antiglide plate (Fig. 1).

Radiological results after 7.1 years in relation to the presence and the size of the fragment

Grade 1 arthroses were seen in some fractures with or without shell-like fragments (Fig. 2). Small fragments (not fixed) were present in the majority of cases with a grade 1 arthrosis, even when the reduction was spontaneously anatomical. Fragments that were larger than one-quarter of the articular surface usually exhibited a grade 1 or 2 arthrosis. Grade 3 arthroses were observed in the group where the Volkmann triangle was fixed (one of which was fixed with a slight step-off of $1 \mathrm{~mm}$, while the other was fixed anatomically), but also in one patient with no Volkmann fragment at all!

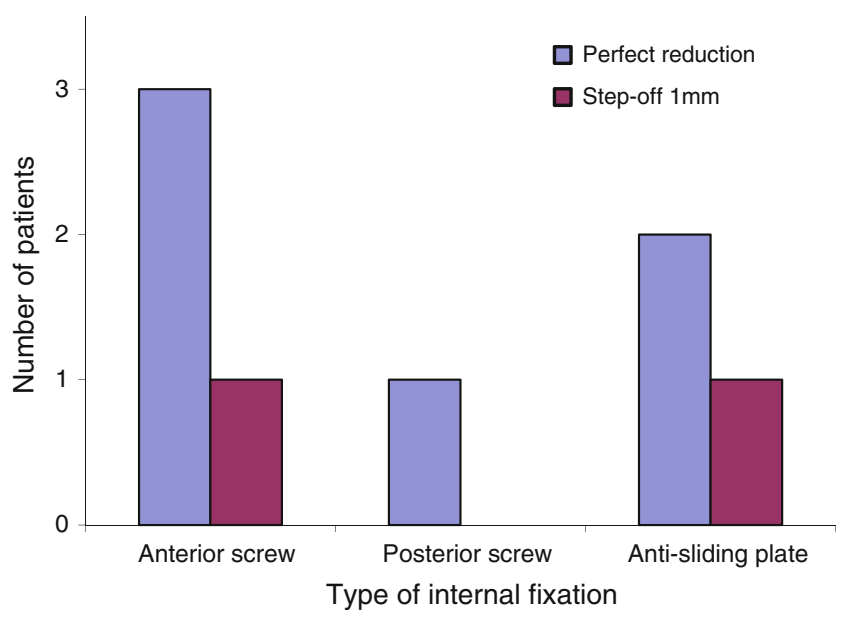

Fig. 1 Mode of internal fixation of Volkmann triangles that were larger than one-quarter of the articular surface, and immediate results of reduction

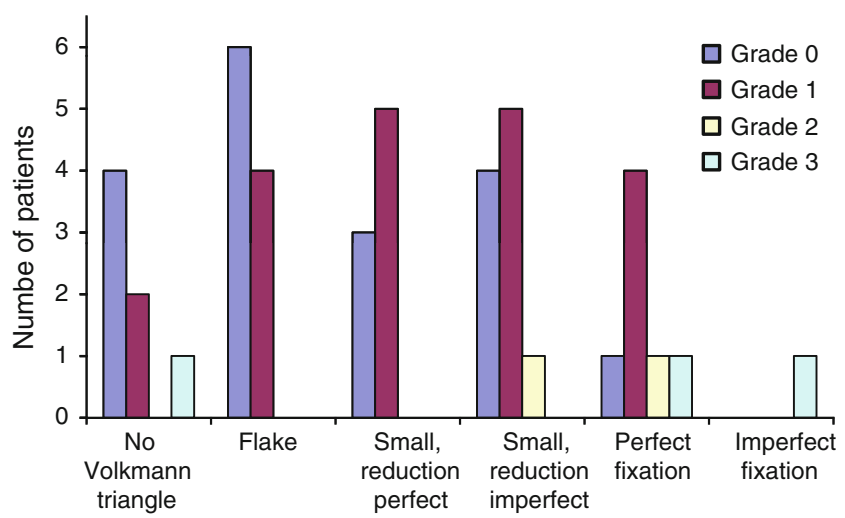

Fig. 2 Grade of arthrosis in relation to the size of the Volkmann triangle after 7.1 years
Clinical results after 7.1 years in relation to the size of the Volkmann fragment

The Olerud-Molander score [13] was used to assess clinical results. The score for patients without a Volkmann triangle was 95.5, in the group with only a small fragment the score was 98.5, and in the group with a fixed Volkmann triangle it was 90.6. This difference was not statistically significant ( $p=0.09$ /one-way ANOVA).

Radiological results after 7.1 years in relation to the presence of a cartilage lesion

The 27 patients without cartilage damage showed better radiological results: they presented mostly no or only grade 1 arthrosis; just 1 patient had a grade 2 arthrosis (Fig. 3). Patients with cartilage damage upon internal fixation [16] mostly presented arthroses of grades $1-3$ !

Clinical results after 7.1 years in relation to cartilage damage

Again, the Olerud-Molander score [13] was used to assess clinical results. In the presence of cartilage damage the score was 94.5, versus 97.2 without any visible damage, although this difference is not statistically significant ( $p=0.8 /$ Student's $t$ test).

\section{Discussion}

Dislocated malleolar fractures are very severe lesions. There are very few large, long-term series for these fractures: either the follow-up is rather short $[5,14]$ or long-

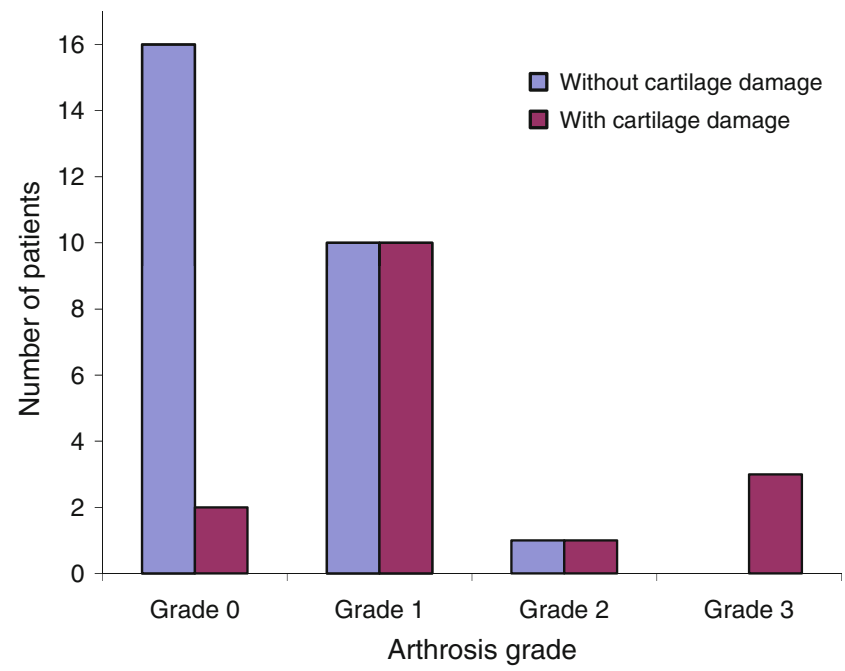

Fig. 3 Grade of arthrosis in relation to cartilage damage after 7.1 years 
term results are derived from only small groups of patients [15-17]. Our follow-up rate of $76 \%$ is just within the the limit of acceptance, and may be explained by the fact that quite a lot of our patients are winter tourists from abroad.

Our small series is not conclusive regarding the development of an arthrosis, and is also not conclusive regarding the clinical outcome in the clinical subjective judgement of the patient in relation to the presence or size of the Volkmann triangle. This may be because the number of patients was just too small. On the other hand, the results clearly reflect the recent uneasiness in the literature over the 70year-old dogma for the treatment of the Volkmann triangle.

It is presently believed that three factors play important roles in the development of arthrosis in malleolar fractures:

1. A change in the contact stresses of the joint. It was demonstrated experimentally that a lateral talus shift of $1 \mathrm{~mm}$ produces a decrease in the contact area of $42 \%$ $[18,19]$, which underlines the importance of the lateral malleolus, considering that "the talus not only goes with the lateral malleolus, but also stays with it" [20]. In relation to the assumed reduction in contact area in the presence of a Volkmann triangle, statistical experimental studies have shown that the larger the Volkmann triangle, the greater the change in the contact area [7, 9]. However, these results are currently heavily contested in the light of the results of recent dynamic/kinematic experimental stress analyses [21].

2. Instability of the ankle mortise with the presumed tendency for subluxation in the presence of a Volkmann triangle that is larger than one-third of the articular surface $[8,9]$.

3. Joint incongruity due to the fractured Volkmann triangle, which leads to an increase in the load on the adjoining joint surfaces [7].

The fixation of Volkmann fragments that are larger than one-quarter of the articular surface was advocated because of these reasons. However, some doubt was shed on these assumptions a few decades ago due to the fact that similar clinical outcomes were obtained for malleolar fractures with a big Volkmann fragment whether or not it was fixed [20]. Later, an experimental investigation using photoelastic models revealed that the load on the posterior surface of the distal tibia was indeed very low and present only in dorsiflexion [22]. Then, in 2004, Fitzpatrick [21] used a dynamic experimental setup to demonstrate that the role commonly attributed to the Volkmann triangle in ankle fractures was doubtful: in their model, four fracture configurations for the posterior malleolus were mimicked (while the internal and external malleolus were kept intact, so that the setup resembled a stable, perfectly reduced situation with a lateral and medial malleolar fracture): an intact ankle mortise, a Volkmann triangle corresponding to
$50 \%$ of the articular surface, the same size (mal) of Volkmann triangle that had been fixed with a $2 \mathrm{~mm}$ gap and a $2 \mathrm{~mm}$ step-off, and the same size of Volkmann triangle that had been anatomically reduced and fixed. In all configurations there was a shift in the location of the contact stresses to the anterior and medial parts of the joint, indicating that this shift causes a part of the ankle joint to receive a big load when this cartilage normally does not have to take such a load. However, most intriguing was the fact that, despite the anatomical reduction and fixation of a big Volkmann triangle, no return to the normal situation of an intact ankle joint was observed. Furthermore, in none of these configurations was a tendency for subluxation noted. Fitzpatrick's experimental data are supported and have been confirmed by van Bekerom's systematic review of the literature, including clinical and biomechanical publications [23]. No differences in outcome were found whether Volkmann fragments that were bigger than one-third of the joint surface were reduced and fixed or just left alone. This confirms Broos' findings [5]: that the prognosis of a malleolar fracture is always compromised by the presence of a fracture of the posterior malleolus. It also challenges Jaskulka's finding [3] that the prognosis of a malleolar fracture improves when a large Volkmann triangle is reduced and fixed. The results of our small series show (though without statistical significance) that there is a trend towards impaired prognosis in the presence of a Volkmann fracture, even when it is correctly fixed. On the other hand, cartilage damage clearly leads to worse results, but such damage cannot be repaired by any surgical maneuver.

\section{Conclusion}

The outcome of a fracture with a Volkmann triangle seems to be fated, whatever the surgeon does (under the condition that the lateral and medial sides are perfectly reduced and fixed and the joint is stable). Also, cartilage damage may be present from the beginning of the trauma, and this may determine the clinical outcome without any chance for the surgeon to intervene. In fact, of the three issues that influence the outcome (see the "Introduction"), two apparently cannot be influenced by the surgeon. The only one that remains is instability! Instability is tolerated in different ways in the joints of the lower extremities. The ankle joint does not tolerate it well [24], and instability can be influenced by the surgeon. Indeed, it is mandatory to test ankle stability after performing fixation of the lateral and medial sides. If there is still instability in the presence of a Volkmann triangle, the fragment must be fixed. Fixing the Volkmann triangle is a better option than fixing the ankle mortise with a positioning screw [25]. 
The Volkmann dogma has guided us through many decades, but it looks as if we have followed a phantom!

Conflict of interest None.

\section{References}

1. Nelson MC, Jensen NK. The treatment of trimalleolar fractures of the ankle. Surg Gynec Obstet. 1940;71:509-14.

2. Mc Laughlin HL. Trauma. Philadelphia: WB Sanders; 1959.

3. Jaskulka RA, Ittner G, Schedl R. Fractures of the posterior tibial margin: their role in the prognosis of malleolar fractures. J Trauma. 1989;29:1565-70.

4. Graff U. Die Bedeutung des Volkmannschen Dreieckes im Hinblick auf die behandlung und Prognose der Knöchelbrüche. Langenbecks Arch Klin Chir Ver Dtsch Z Chir. 1954;279: 809-15.

5. Broos PLO, Bisshop APG. Operative treatment of ankle fractures in adults: correlation between types of fracture and final results. Injury. 1991;22:403-6.

6. de Souza LJ, Gustilo RB, Meyer TJ. Results of operative treatment of displaced external rotation-abduction fractures of the ankle. J Bone Joint Surg A. 1985;67(7):1066-73.

7. Hartford JM, Gorczyca JT, McNamara JL, Mayor MB. Tibiotalar contact area. Contribution of posterior malleolus and deltoid ligament. Clin Orthop. 1995;320:182-7.

8. Maatz R. Die Fixation des hinteren Volkmann-Dreiecks mit der Federkopfschraube. Bruns Beitr Klein Chir. 1963;207:433-42.

9. Macko VW, Matthews LS, Zwirkoski P, Goldstein SA. The jointcontact area of the ankle. J Bone Joint Surg A. 1991;73(3): 347-51.

10. Knirk JL, Jupiter JB. Intraarticular fractures of the distal end of the radius in young adults. J Bone Joint Surg A. 1986;68:647-59.

11. Weber BG. Die Verletzungen des oberen Sprunggelenkes. Bern: Hans Huber; 1966.
12. Gustilo RB, Anderson JT. Prevention of infection in the treatment of one thousand and twenty-five open fractures of long bones. J Bone Joint Surg A. 1976;58(4):453-8.

13. Olerud C, Molander H. A scale for symptom evaluation after ankle fractures. Arch Orthop Trauma Surg. 1984;103:190-4.

14. Lindsjö U. Operative treatment of ankle fracture-dislocations. Clin Orthop Rel Res. 1985;199:28-38.

15. Specchiulli F, Mangialardi R. The surgical treatment of malleolar fractures: long-term results. Chir Organi Mov. 2004;89(4):313-8.

16. Joz-Roland P, Kritsikis N, Cyprien JM. Long term results of treatment of malleolar fractures. Rev Chir Orthop Reparatrice Appar Mot. 1980;66(3):173-82.

17. Day GA, Swanson CE. Operative treatment of ankle fractures. A minimum ten-year follow-up. Foot Ankle Int. 2001;22(2):102-6.

18. Ramsey P, Hamilton W. Changes in tibiotalar contact caused by talar shift. J Bone Joint Surg A. 1976;58:356-7.

19. Lloyd J, Elsayed S, Hariharan K, Tanaka H. Revisiting the concept of talar shift in ankle fractures. Foot Ankle Int. 2006;27(10):793-6.

20. Harper MC, Hardin G. Posterior malleolar fractures of the ankle associated with external rotation-abduction injuries. J Bone Joint Surg A. 1988;70(9):1348-56.

21. Fitzpatrick DC, Otto JK, McKinley TO, Marsh JL, Brown TD. Kinematic and contact stresses analysis of posterior malleolus fractures of the ankle. J Orthop Trauma. 2004;18(5):271-8.

22. Papachristou G, Efstathopoulos N, Levidiotis C, Chronopoulos E. Early weight bearing after posterior malleolar fractures: an experimental and prospective clinical study. J Foot Ankle Surg. 2003;42(2):99-104.

23. van den Bekerom MPJ, Haverkamp D, Kloen P. Biomechanical and clinical evaluation of posterior malleolar fractures. A systematic review of the literature. J Trauma. 2009;66:279-84.

24. McKinley TO, Rudert MJ, Koos DC, Brown TD. Incongruity versus instability in the etiology of posttraumatic arthritis. Clin Orthop. 2004;423:44-51.

25. Gardner MJ, Brodsky A, Briggs SM, Nielson JH, Lorich DG. Fixation of posterior malleolar fractures provides greater syndesmotic stability. Clin Orthop. 2006;447:165-71. 